\title{
Functional Analysis of the Vertebral Column Based on MR and Direct Volume Rendering
}

\author{
P. Hastreiter ${ }^{1}$, C. Rezk-Salama ${ }^{2}$, K. Eberhardt ${ }^{3}$ and B. Tomandl ${ }^{3}$, and T. Ertl ${ }^{4}$ \\ 1 Neurocenter, University of Erlangen-Nuremberg, Germany \\ hastreiter@nch.imed.uni-erlangen.de \\ 2 Computer Graphics Group, University of Erlangen-Nuremberg, Germany \\ 3 Division of Neuroradiology, University of Erlangen-Nuremberg, Germany \\ 4 Visualization and Interactive Systems Group, University of Stuttgart, Germany
}

\begin{abstract}
Degenerative diseases of the vertebral column are mainly combined with misalignments of the intervertebral discs and deformations of the spinal cord. For the investigation of severe spinal stenosis and herniated intervertebral discs $\mathrm{x}$-ray myelography is required providing important functional information about the motion of vertebral segments. To avoid this time-consuming procedure and the injection of contrast agent, we introduce a non-invasive approach using direct volume rendering of a novel T2 weighted MR sequence. It provides meaningful representations of extreme spinal pathologies. Above all, the essential functional information is obtained using an adjustable couch directly applied within the MR scanner. Due to the distribution of the grey values, we suggest a coarse segmentation of the vertebral column using a fast sequence of filtering operations and volume growing. Further on, details are delineated implicitly using interactive adjustment of transfer functions. Thereby, the CSF, the roots of the spinal nerves and the vertebrae are clearly visible. Additionally, we propose an approach for volumetric measurement providing the functional dependence of the CSF volume. The presented non-invasive strategy gives a comprehensive understanding of spatial and functional information including spinal instability and may thus lead to further reduce the need of x-ray myelography.
\end{abstract}

Keywords: Visualization, Functional Analysis, Spine, Therapy Planning

\section{Introduction}

Degenerative discogenic diseases of the vertebral column are mainly caused by misalignments of the intervertebral discs and deformations of the spinal cord. For their investigation a clear representation of the spinal cord in relation to the roots of the nerves and the surrounding bone structures is required. For these examination there has been a great change of diagnostic methods. This is mainly related to the enormous development of magnetic resonance (MRI) and x-ray computed tomography $(C T)$. Above all, MRI gains of increased importance since the parameters of the imaging sequence can be optimized according to the application and the applied post-processing procedure [1]. As a further advantage of tomographic imaging techniques three-dimensional $(3 D)$ image data is produced which provides spatial understanding. However, severe cases such as 
extreme spinal stenoses and herniated intervertebral discs with unconfirmed root compressions still require to perform $x$-ray myelography. So far, due to its detailed imaging capabilities it is the only imaging approach giving an exact correlation of the roots of the spinal nerves, the vertebral segments and surrounding bone structures. Further on, it provides valuable functional information which is essential for a comprehensive examination. This is of great importance since the location of vertebral segments and the representation of the spinal cord changes considerably during motion. However, as major drawback the injection of a non-ionic contrast agent is required which is applied intrathecally. In general four standard projections are taken with anterior-posterior, lateral and left-anterior-oblique (LAO) or right-anterior-oblique (RAO) orientation. This is a time-consuming procedure which is also combined with a certain risk.

The idea of the presented approach is to introduce a new strategy for a comprehensive analysis of discogenic diseases within the vertebral column based on MR image data exclusively. After a short overview in section 2 explaining the main issues of the applied image data, a newly developed MR compatible couch is discussed in section 3 which allows to position the spine. Then, section 4 demonstrates which anatomical structures have to be separated explicitly and how to segment them efficiently. In order to visualize the available 3D image data comprehensively an appropriate visualization approach is indispensable. Therefore, we suggest to use interactive direct volume rendering which allows to show all available information including vertebral instability. The applied hardware accelerated approach is briefly illustrated in section 5. It is also explained how to efficiently achieve a meaningful representation of the vertebral segments, the spinal cord and the roots of the spinal nerves. Additionally, in case of an arteriovenous malformation a fusion strategy is introduced which allows to integrate a representation of dural vessel information as presented in [2]. Since volumetric information is of major interest section 6 presents an approach which allows to analyze the functional dependence of the CSF volume. Finally, section 7 presents several clinical examples demonstrating the value of the suggested approach.

\section{Image Data}

The advances of MR scanning techniques produced a variety of special MR sequences which give more detailed insight to the different structures of the vertebral column. As a basis of our approach MEDIC (Multi Echo Data Image Combination) data is applied which is a strongly T2-weighted MR sequence. It clearly distinguishes the CSF, the vertebral discs and the roots of the spinal nerves. As demonstrated in figure 1 (left) they are mapped to high intensities whereas bone structures have low intensities. Since parts of the surrounding tissue is in the same range of data values simple visualization strategies like maximum intensity projection $(M I P)$ provide insufficient $3 \mathrm{D}$ representations. Contrary to that explicit segmentation of the image data is difficult due to partial volume effects. Therefore, interactive direct volume rendering is applied based on its capability to perform implicit segmentation using transfer functions.

As an advantage over the FISP (Fast Imaging with Steady State) sequence which was previously [1] evaluated using two-dimensional (2D) visualization, the MEDIC sequence allows to perform measurements with an improved in-slice resolution. Thereby, 
smaller intra-dural structures become visible much better and any post-processing like segmentation or volume visualization is considerably simplified. As presented in [2], MR-CISS (Constructive Interference in the Steady State) data, shown in figure 1 (right), is optimally suited to detect structures within the dura. Thereby, tiny vessel malformations are clearly distinguished from CSF. Contrary to that, the applied MEDIC sequence hardly delineates these vessel structures. However, it optimally shows the intervertebral discs and differentiates the CSF from epidural fat.
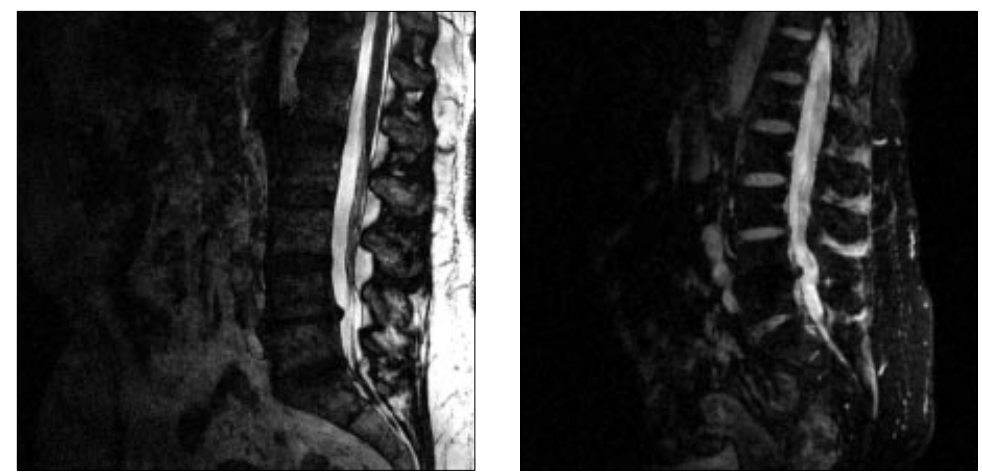

Fig. 1. MR-CISS (left) and MR-MEDIC (right) difference of bone structures, discs and CSF.

\section{Functional Analysis}

Functional information about the motion dependence of the vertebral column represents a fundamental prerequisite for the investigation of degenerative discogenic diseases. Using an adjustable couch which is applied directly in a standard MR scanner, different positions are achieved simulating the movement of the vertebral column. Depending on the state of the disease a "normal" position is defined for every individual patient according to the normal position used for standard MRI scans. This is in correspondence with the system described in [3]. Then, using the device the patient is successively lifted at the head and at the back in order to perform inclination and reclination relative to the normal position. Thereby, changes of the vertebrae and the spinal cord are clearly detected allowing for geometric and volumetric assessment. As major advantage of this approach, it is applicable with an arbitrary MR scanner. Further on, the couch gives the necessary assistance which is essential for older patients or in case of severe diseases.

Other approaches suggesting an investigation with open MR scanners have severe disadvantages. The main drawback is the restricted availability of these systems since they are installed at very few sites. Due to the low magnetic field of such scanners only image data of lower resolution is provided. However, high resolution is essential for the analysis of intra-dural structures such as the roots of the spinal nerves and for volumetric measurements related to the CSF. Above all, the lack of an assisting device supporting the movement of the patient is the most important drawback. Thereby, longer scanning times are prohibited and older patients or patients with extreme spinal pathologies are excluded from an examination. 


\section{Explicit Segmentation}

According to section 2 the MR-MEDIC shows high contrast between CSF and surrounding tissue. However, both the CSF and the intervertebral discs are in the same range of intensity values. This makes an implicit delineation difficult using a lookup table which affects the data globally. Therefore, explicit segmentation is performed with fast filtering and volume growing (see figure 2). Thereby, the CSF including the roots of the spinal nerves and the vertebrae are clearly separated.

Initially, noise reduction is performed to optimize the data for further pre-processing. Successively, the CSF including the nerve roots is extracted by semi-automatic volume growing. As a basis of this strategy flexible bounding boxes are applied which are interactively adjusted to the underlying anatomy. Thereby, minimal post-processing is required and the target structure is optimally separated. In the same way the area is isolated which includes the bone structures and the intervertebral discs.

Based on the segmentation results, the original image data is attributed using unique tag numbers for the CSF including the nerve roots, the bone area and the surrounding tissue. Thereby, separate transfer functions are assigned which locally manipulate the look-up tables for color and opacity values. Due to the immediate visual control and the low computational expense this strategy is much faster and more robust than an explicit segmentation of details which might easily miss important features.
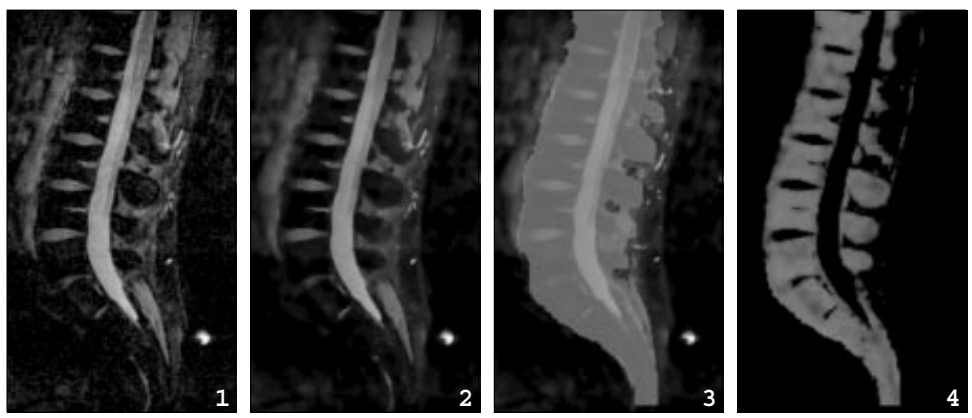

Fig. 2. Segmentation of MR-MEDIC: (1) original image, (2) noise reduction , (3) volume growing for the separation of CSF and bone structures, (4) inversion of segmented bone structures.

\section{Visualization}

For the analysis of medical image data it is indispensable to choose an appropriate visualization approach. Compared to indirect strategies [4] which rely on polygonal representations, direct volume rendering has proved to be superior [5]. Since the information of all data samples is used, image characteristics like partial volume effects are better taken into account. However, interactive manipulation is an essential feature for an application in a clinical environment [6].

All visualizations of the presented approach are based on direct volume rendering using 3D-texture mapping [7]. The basic idea of the algorithm is to define the image 

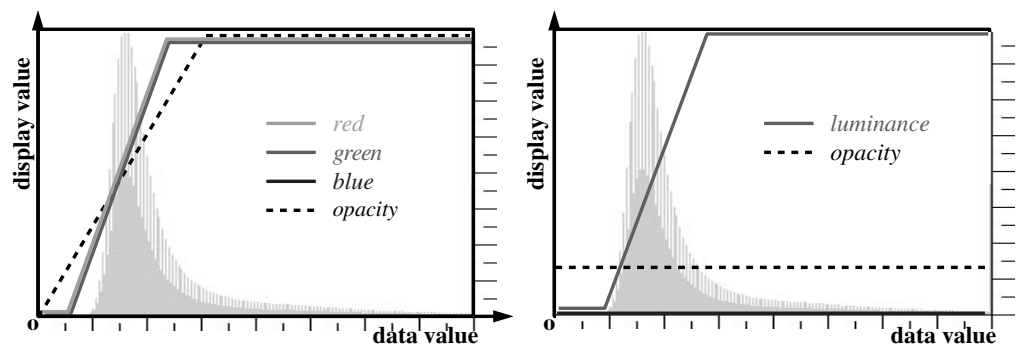

Fig. 3. Intensity histogram and transfer functions for Figure 6: (left) Setting for the CSF including the roots of the spinal nerves — (right) Setting applied to the background.

data as a 3D-texture. Then, equidistant slices parallel to the image plane are cut out of the volume. Thereby, the texture mapping hardware is exploited by performing trilinear interpolation in order to reconstruct every slice. Finally, the $3 \mathrm{D}$ representation is obtained by blending the textured slices back-to-front onto the viewing plane.

As an advantage of this approach, interactive manipulation of any viewing parameter is achieved even if applied to volumes of high resolution. Due to the applied trilinear interpolation scheme and the number of sampling points, which are appropriately adjusted, the resulting images are of high quality. This guarantees to reproduce fine structures and ensures their clear delineation.

Using the segmentation of the MEDIC data as described in section 4, detail information is delineated by adjusting the respective transfer functions. For every tagged subvolume four separate curves are used describing the correlation between the original data values and the displayed color components (RGB) and opacity. In order to speed up the process of adjusting these transfer functions, pre-defined templates are used.

In Figure 3 the setting of the transfer functions for color and opacity values is shown which leads to the visualization presented in Figure 6. Although arbitrary transfer functions are applicable, a piecewise linear mapping is sufficient. The visualization of the CSF (tag 1) requires to use low opacity values for low data values and high opacity values for high data values. Further on, a linear ramp of high gradient is used in between in order to produce a smooth transition. The transfer function for color values is positioned within the transition from low to high opacity values. This leads to a good impression of depth and enhances the representation of the nerve roots. To reveal the vertebrae and the intervertebral discs ( $\operatorname{tag} 2)$ the opacity is set to its maximum. Just for low data values a steep linear ramp is applied in order to suppresses artifacts remaining from the coarse segmentation. This results in a fully opaque representation which supports the anatomical orientation. For the background region (tag 3 ) the opacity is set to full transparency since there is no further information of importance.

\section{Volumetric Measurement}

The MR compatible device discussed in section 3 allows to investigate different positions of the vertebral column: normal position, inclination, reclination. Depending on 
the selected position the representation of the CSF volume changes. Therefore, knowledge of this functional dependence is of major interest for the examination. An essential prerequisite for this volumetric measurement is the explicit segmentation of the CSF as presented in section 4. Including the roots of the spinal nerves this is easily achieved with MR-MEDIC data due to the clear delineation from surrounding structures.

With respect to denerative discogenic diseases of the vertebral column it is important to assess the functional dependence of the CSF volume in the vicinity of the respective intervertebral discs. Therefore, as shown in figure 4, a strategy is suggested which allows standardized measurements using a cylindrical bounding box. Having set its extent to a user defined value, the bounding box is applied to the volume data of all functional states which are investigated. Additionally, within each data set the bounding box is rotated appropriately. Using its centerline as a reference it is then centered at the selected intervertebral disc. As a result a subvolume of the segmented CSF including the nerve roots is specified which is compared to the volume of the other positions.

According to section 2 the CISS sequence differentiates between CSF and the roots of the spinal nerves. In contrast to that both structures are represented as a unique volume with MR-MEDIC. However, as presented in figure 5, the combined volume of the $M E D I C$ data is identical to the sum of the subvolumes obtained with CISS data. In consequence there is a clear dependence of the CSF volume and the volume of the roots exiting a specific segment as well as the respective parts of the descending roots. Therefore, the $M E D I C$ sequence is sufficient to evaluate the degree of compression. This was confirmed by neurophysiological examinations with 20 patients.

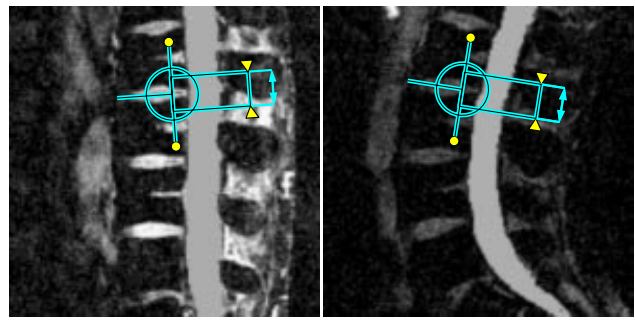

Fig. 4. Volumetric measurement.

\begin{tabular}{|c|c|c|c|}
\hline Position & $\begin{array}{c}\text { CSF +roots } \\
(M E D I C)\end{array}$ & $\begin{array}{c}\text { roots } \\
(\text { CISS })\end{array}$ & $\begin{array}{c}\text { CSF } \\
(\text { CISS })\end{array}$ \\
\hline inclinat. & 40374 & 10750 & 29624 \\
\hline normal & 44773 & 10488 & 34295 \\
\hline reclinat. & 35325 & 11562 & 23763 \\
\hline
\end{tabular}

Fig. 5. Volumetric measurements in $\mathrm{mm}^{3}$.

\section{Results and Discussion}

So far, the presented approach was applied to 50 healthy volunteers and 80 patients with severe spinal stenosis and spondylo listeses. In all cases the diagnosis was performed in comparison with $\mathrm{x}$-ray myelography. All MR-MEDIC volumes were acquired with a Siemens MR Magnetom Symphony 1.5 Tesla scanner which provides the necessary resolution to resolve the relevant structures appropriately. In all cases volumes were used consisting of images with a $256^{2}$ matrix and 30-50 slices. The size of the voxels was set to $1.17 \times 1.17 \times 1.17 \mathrm{~mm}^{3}$. In order to achieve high frame rates, all direct vol- 
ume rendering was performed with a SGI Onyx2 (R10000, 195MHz) with BaseReality graphics hardware providing $64 \mathrm{MB}$ of texture memory.

In all cases the investigation was performed with a standardized protocol. Using the MR-MEDIC sequence three scans were performed showing the normal position, inclination and reclination of the vertebral column. On the basis of this image data, direct volume rendering was performed of every data set. In order to give a good overview of the lesion, the selected directions of view comprise posterior-anterior, oblique posterior and lateral. The following examples demonstrate the value of the presented approach:

- In figure 6 the image data of a healthy volunteer is shown. The example demonstrates the strategy of the applied visualization technique and illustrates the capabilities of the presented approach with respect to intra-dural substructures such as the roots of the spinal nerves.

- The case of a multi segment stenosis in levels L2-L5 is presented in figure 7. Applying direct volume rendering the degree of the lesion is precisely determined in all levels. Due to osteochondrosis disc L5/S1 is not visible. The comparison of inclination and reclination illustrates the extremely restricted range of movement which is the case for the majority of older patients.

- The example of figure 8 shows a multi segment spinal stenosis. Using X-ray myelography the contrast agent stops completely at the stenosis just after intrathecal application (a). About 30 minutes later the situation changes completely after the contrast agent has past the stenosis $(b, d)$. However, the representation of the lesion prohibits to determine its exact level which is essential information to minimize the risk of surgery. In contrast, direct volume rendering of MR-MEDIC data (c,e,f) clearly shows the location of all segments with different directs of view. This allows to evaluate the compression of the nerve roots at every level.

- In figure 9 the compression of both nerve roots in level L5 is presented. Based on an additional segmentation of the vertebrae a semi-transparent visualization tremendously assists the investigation of the lesion from all directions. The aliasing artifacts within the lateral view are caused by a sub-optimal field of view (FoV). This was required to minimize the time of examination to prevent breathing artifacts.

- Figure 10 shows the functional dependence of the vertebral column in case of a healthy volunteer. Direct volume rendering was performed after explicit segmentation of the bone structures.

As demonstrated in these examples, our strategy based on non-invasively acquired MR-MEDIC data allows to analyze degenerative diseases of the vertebral column in a comprehensive way. Compared to the normal position, the CSF and the intervertebral discs are better delineated during inclination of the vertebral column. Additionally, any instability and movement of vertebral segments are clearly represented with the functional image data. In all cases with extreme spinal stenosis and a reduced CSF volume of more than $70 \%$ the level of the lesion was clearly determined. Contrary to that x-ray myelography prohibited a secure determination in these cases.

Due to the distribution of the grey values and the complexity of the target structures, the explicit segmentation of the CSF including the roots of the spinal nerves and the surrounding vertebrae requires a time-consuming process. Therefore, the approach based 
on filtering and semi-automatic volume growing provides a more convenient and robust way for segmentation. Since the structures are clearly delineated within the image data, this process takes approximately 5-10 minutes. For the successive direct volume rendering of the attributed volume interactive adjustment of transfer functions is important. Thereby, direct visual feedback is guaranteed. Based on pre-defined lookup tables for color and opacity values, the adjustment of the respective transfer functions takes another 5 minutes. Applying 3D texture mapping, the trilinear interpolation scheme provides excellent image quality and the hardware acceleration ensures high frame rates.

\section{Conclusion}

The presented approach introduces a non-invasive strategy in order to investigate severe degenerative diseases of the vertebral column. Using MR-MEDIC images the important structures are clearly delineated. Thereby, only the CSF including the roots of the spinal nerves and in selected cases the bone structures have to be segmented explicitly. This allows convenient volumetric measurements for a more detailed analysis of the lesion. A detailed representation of the volume data is then obtained using implicit delineation on the basis of direct volume rendering. The essential functional information about the motion dependence of the vertebral column is obtained with a MR compatible device. The presented clinical examples demonstrate the value of our approach. In consequence, the pre-operative investigation of severe degenerative diseases within the vertebral column is effectively assisted and the need of x-ray myelography is considerably reduced.

\section{References}

[1] K. Eberhardt, H. Hollenbach, B. Tomandl, and W. Huk. Three-Dimensional MR myelography of the lumbar spine: comparative case study to X-ray myelography. In European Radiology, volume 7, pages 737-743, 1997.

[2] C. Rezk-Salama, P. Hastreiter, K. Eberhardt, B. Tomandl, and T. Ertl. Interactive Direct Volume Rendering of Dural Arteriovenous Fistulae. In Proc. MICCAI'99 (2nd Int. Conf on Med. Img. Comput. and Comp.-Assis. Interv.), volume 1679 of Lect. Notes in Comp. Sc. 1679, pages 42-51. Springer, 1999.

[3] J. Dvorak and M. Panjabi et al. Functional radiographic diagnosis of the lumbar spine. SPINE, 16:562-571, 1991.

[4] S. Nakajima, H. Atsumi, A. Bhalerao, F. Jolesz, R. Kikinis, T. Yoshimine, T. Moriarty, and P. Stieg. Computer-assisted Surgical Planning for Cerebrovascular Neurosurgery. Neurosurgery, 41:403-409, 1997.

[5] B. Kuszyk, D. Heath, D. Ney, D. Bluemke, B. Urban, T. Chambers, and E. Fishman. CT Angiography with Volume Rendering : Imaging Findings. American Jour. of Radiol. (AJR), pages 445-448, 1995.

[6] L. Serra, R. Kockro, C. Guan, N. Hern, E. Lee, Y. Lee, C. Chan, and W. Nowinsky. Multimodal Volume-Based Tumor Nerurosurgery Planning in the Virtual Workbench. In Proc. Med. Img. Comput. and Comp.-Assis. Interv. (MICCAI), volume 1496 of Lec. Notes in Comp. Sc., pages 1007-1015. Springer, 1998.

[7] B. Cabral, N. Cam, and J. Foran. Accelerated Volume Rendering and Tomographic Reconstruction Using Texture Mapping Hardware. ACM Symp. on Vol. Vis., pages 91-98, 1994.

Note to reviewers: the color pages can also be found at http://www9.informatik.uni-erlangen.de/eng/research/vis/Spine/miccai.html 

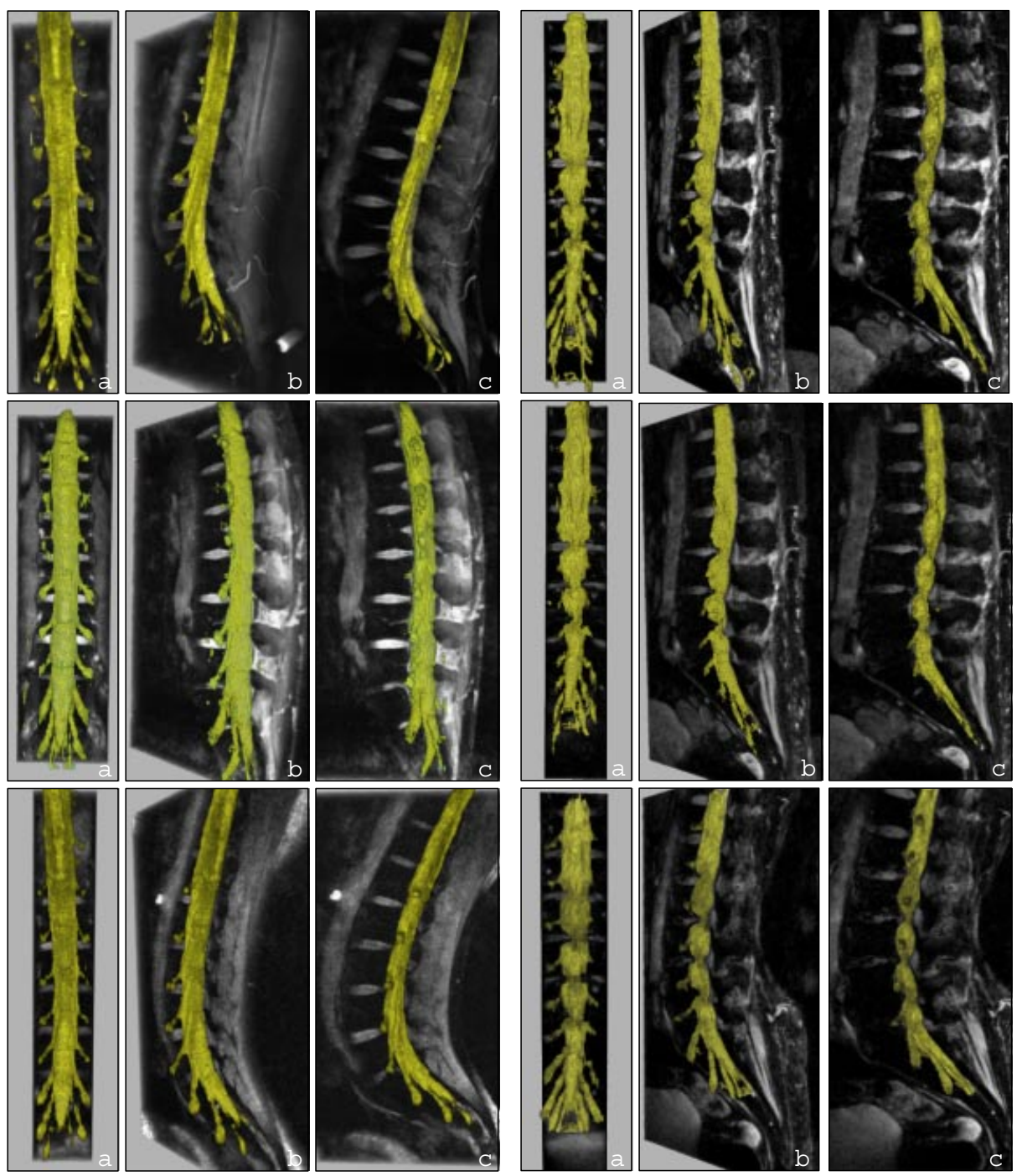

Fig. 6. Visualization showing normal position (top), inclination (middle) and reclination (bot-

Fig. 7. Visualization showing normal position (top row), inclination (middle row) and reclinatom) of healthy volunteer with standardized views: $(a)$ posterior-anterior, $(b)$ oblique posterior and $(c)$ lateral.

tion (bottom row) of patient with spinal stenosis with standardized views: (a) posterior-anterior, (b) oblique posterior and (c) lateral. 

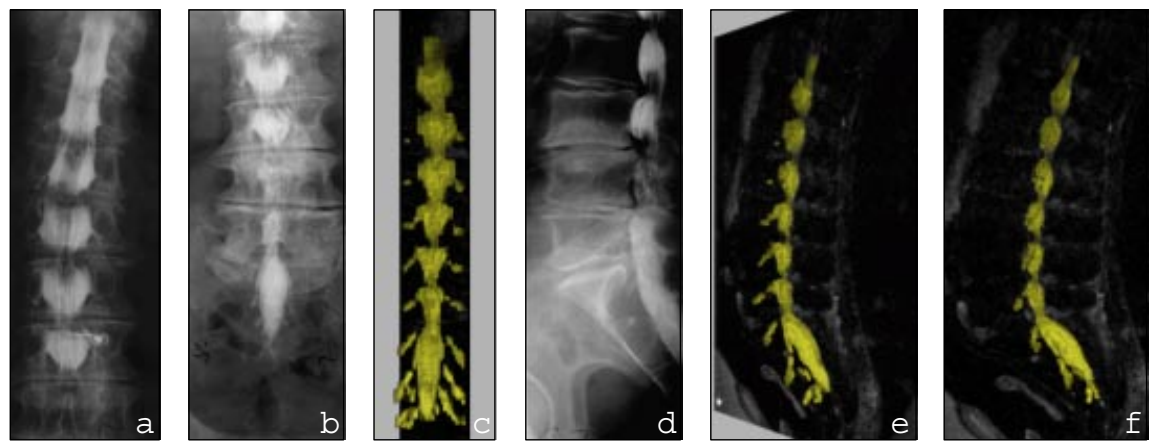

Fig. 8. Patient with multi-segment spinal stenosis: comparison of $x$-ray myelography $(a, b, c)$ and examination based on MR-MEDIC data and direct volume rendering with different directions of view: posterior-anterior $(c)$, oblique posterior $(e)$ and lateral $(f)$.
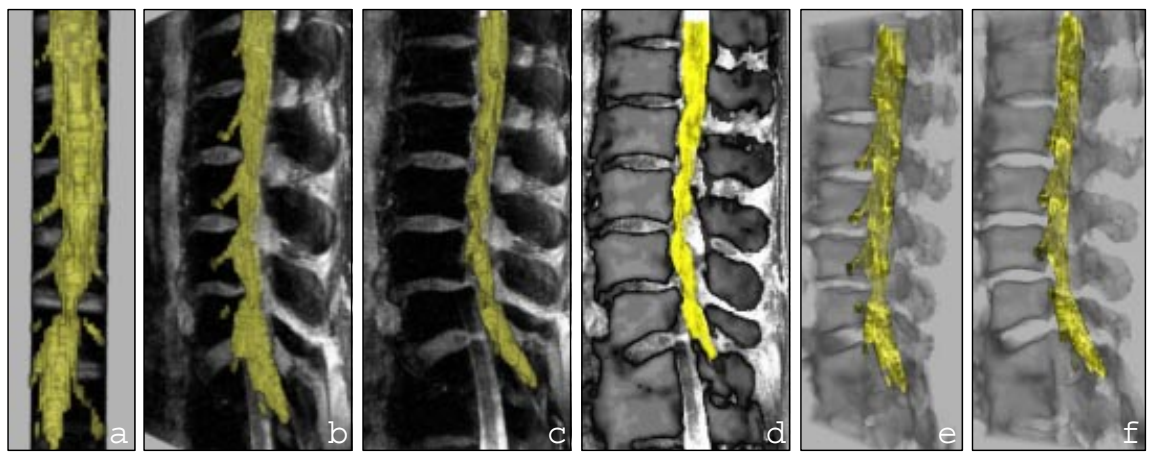

Fig. 9. Direct volume rendering of MR-MEDIC data showing a mono-segment stenosis in combination with a spondylo listesis in L4 and L5: 3D representation without $(a, b, c)$ and with $(d, e, f)$ explicit segmentation of bone structures.
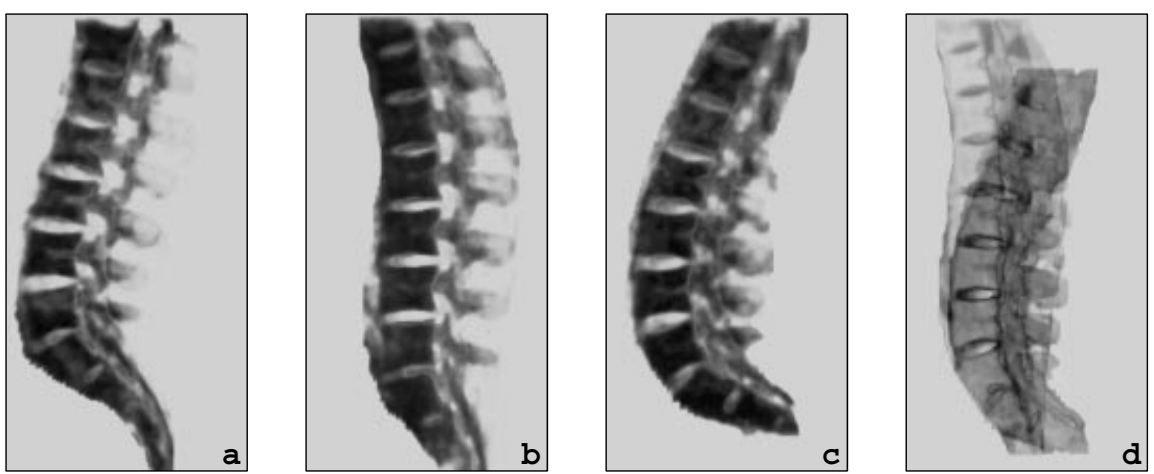

Fig. 10. Direct volume rendering of functional MR-MEDIC data: Healthy volunteer showing the bone structures of the vertebral column: normal position (a), inclination (b), reclination (c) and superposition of inclination and reclination (d). 